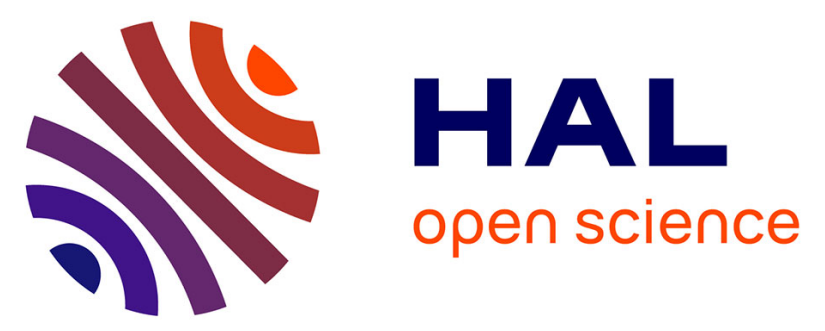

\title{
Etudes sous champ magnétique de l'effet tunnel résonnant et non résonnant dans les structures à double barrière n-(AlGa)As/GaAs
}

\author{
Alain Celeste, J.C. Portal, L. Eaves, E.S. Alves, T.J. Foster, M. L. \\ Leadbeater, G. Hill, M. A. Pate
}

\section{To cite this version:}

Alain Celeste, J.C. Portal, L. Eaves, E.S. Alves, T.J. Foster, et al.. Etudes sous champ magnétique de l'effet tunnel résonnant et non résonnant dans les structures à double barrière n-(AlGa)As/GaAs. Revue de Physique Appliquée, 1989, 24 (3), pp.343-349. 10.1051/rphysap:01989002403034300 . jpa00246056

\section{HAL Id: jpa-00246056 https://hal.science/jpa-00246056}

Submitted on 1 Jan 1989

HAL is a multi-disciplinary open access archive for the deposit and dissemination of scientific research documents, whether they are published or not. The documents may come from teaching and research institutions in France or abroad, or from public or private research centers.
L'archive ouverte pluridisciplinaire HAL, est destinée au dépôt et à la diffusion de documents scientifiques de niveau recherche, publiés ou non, émanant des établissements d'enseignement et de recherche français ou étrangers, des laboratoires publics ou privés. 
Classification

Physics Abstracts

$73.40 \mathrm{Gk}-72.80 \mathrm{Ey}-72.20 \mathrm{My}-73.20 \mathrm{Dx}$

\title{
Etudes sous champ magnétique de l'effet tunnel résonnant et non résonnant dans les structures à double barrière n-(AlGa)As/GaAs
}

\author{
A. Céleste $\left({ }^{1}\right)$, J. C. Portal $\left({ }^{1}\right)$, L. Eaves $\left({ }^{2}\right)$, E. S. Alves $\left({ }^{2}\right)$, T. J. Foster $\left({ }^{2}\right)$, M. L. Leadbeater \\ $\left({ }^{2}\right)$, G. Hill $\left({ }^{3}\right)$ and M. A. Pate $\left({ }^{3}\right)$ \\ (1) Département de Génie Physique, Institut National des Sciences Appliquées, 31077 Toulouse, et SNCI- \\ CNRS, 38042 Grenoble, France \\ (2) Department of Physics, University of Nottingham, Nottingham, NG7 2RD, Grande-Bretagne \\ $\left({ }^{3}\right)$ Department of Electronic and Electrical Engineering, University of Sheffield, Mappin Street, Sheffield S1 \\ 3JD, G.B.
}

(Reçu le $1^{\text {er }}$ juillet 1988, révisé le 3 octobre 1988, accepté le 23 novembre 1988)

\begin{abstract}
Résumé. - Nous avons étudié les propriétés électroniques d'une série de structures à double barrière, dont la largeur du puits varie de 5 à $6 \mathrm{~nm}$. La bistabilité observée couramment dans les caractéristiques $I(V)$ de ces structures, peut être éliminée par l'adjonction aux bornes du dispositif d'une résistance ou d'un condensateur appropriés. Ceci entrave sérieusement les récentes interprétations de cette bistabilité, en termes d'effet intrinsèque de charges d'espaces. Ces caractéristiques stabilisées nous permettent d'étudier en détail l'effet d'un champ magnétique $\mathbf{B} / / \mathbf{J}$ sur le courant vallée de ces dispositifs, où l'on observe des oscillations dues à l'effet tunnel assisté par l'émission de phonons LO et par les processus de diffusion élastiques.

L'effet d'un champ magnétique $\mathbf{B} \perp \mathbf{J}$ sur les structures à large puits $(60 \mathrm{~nm})$ permet d'observer une transition entre l'effet tunnel sur les états hybrides magnéto-électriques et les états cycloïdaux d'interface quantifiés magnétiquement.
\end{abstract}

\begin{abstract}
The electrical properties of a series of double barrier tunnelling devices with well widths between 50 and $600 \AA$ are investigated. It is shown that the bistability effect in the current-voltage characteristics of a typical resonant tunnelling device can be removed by connecting a suitable capacitance or resistance to the device. These measurements cast serious doubt on the recent interpretation of the bistability as an intrinsic space-charge effect. In the stabilised section of the $I(V)$ curve, at voltages above the main resonant peak, the magnetoquantum oscillations observed with $\mathbf{B} / / \mathbf{J}$ are used to investigate tunnelling assisted by LO phonon emission and by elastic scattering processes. The resonant tunnelling device with well width $600 \AA$ exhibits sixteen regions of negative differential conductivity. The effect of a transverse magnetic field $\mathbf{J} \perp \mathbf{B}$ on the resonances in the $I(V)$ characteristics is investigated. At sufficiently high magnetic field, a transition from tunnelling into electrically quantised states to tunnelling into magnetically quantised states is observed.
\end{abstract}

\section{Introduction.}

La structure à effet tunnel résonnant a considérablement attiré l'attention récemment, comme dispositif ultra-rapide prometteur et comme système dont les propriétés électriques sont contrôlées par un processus quantique fondamental. Dans cet article nous présentons une série d'études sous champs magnétiques des propriétés électriques de ces dispositifs. Les structures, utilisant le système GaAs/GaAlAs furent élaborées à Nottingham par EJM, sur un réacteur Varian Gen II. Des dispositifs montrant plusieurs zones de Résistance Différentielle Négative (RDN) avec des rapports pic/vallée jusqu'à 3,6/1 à température ambiante et $25 / 1$ à l'hélium liquide furent fabriqués. Nous décrivons trois sujets d'intérêt particulier :

(1) la question de savoir si oui ou non on observe une bistabilité intrinsèque associée à l'accumulation de charges dans le puits ;

(2) la contribution au courant tunnel de processus de diffusion élastiques et inélastiques, que révèle l'analyse des magnéto-oscillations dans les caractéristiques $I(V)$ pour $\mathbf{B} / / \mathbf{J}$;

(3) la transition de l'effet tunnel sur les états 
quantifiés électriquement, à l'effet tunnel sur les états quantifiés magnétiquement en présence d'un fort champ magnétique $\mathbf{B} \perp \mathbf{J}$.

\section{A t'on pu observer la bistabilité intrinsèque ?}

Récemment, Goldman et al. [1] ont rapporté l'observation de bistabilités dans les caractéristiques courant-tension de structures à effet tunnel résonnant. Cette bistabilité intervient dans la région de Résistance Différentielle Négative. Elle fut interprétée comme une propriété intrinsèque due aux effets électrostatiques des charges accumulées dans le puits à la résonance.

Cette conclusion fut controversée par Sollner [2] qui affirma que la bistabilité observée était une propriété commune aux dispositifs faisant intervenir une zone de Résistance Différentielle Négative et qu'elle était due aux oscillations du courant dans le dispositif et le circuit extérieur. Dans ce cas la bistabilité est due à la différence entre les tensions d'amorçage et de désamorçage des phénomènes oscillatoires lorsque l'on balaye la tension appliquée dans le sens croissant ou dans le sens décroissant. La zone d'extension en tension de cette bistabilité dépend alors des paramètres du circuit extérieur ainsi que des caractéristiques intrinsèques du dispositif.

Récemment, nous avons étudié la dépendance en champ magnétique des caractéristiques courant-tension $[3,4]$ de structures à effet tunnel résonnant dans le système $\mathrm{GaAs} / \mathrm{GaAlAs}$. Nos expériences ont confirmé l'accumulation de charges dans le puits aux tensions de résonance. Dans la zone de RDN, les dispositifs faisaient apparaître une bistabilité identique à celle initialement rapportée par Goldman et al. [1].

La structure étudiée est un plot de $100 \mu \mathrm{m}$ de diamètre, la structure étant la suivante dans l'ordre de croissance à partir du substrat $\mathrm{n}^{+} \mathrm{GaAs}$ (structure A) :

(i) $1 \mu \mathrm{m}$ de GaAs, $n=2 \times 10^{17} \mathrm{~cm}^{-3}$ comme couche tampon; (ii) $8,8 \mathrm{~nm}$ de (AlGa)As non dopé, [Al] $=0,33$; (iii) $5,6 \mathrm{~nm}$ de GaAs non dopé; (iv) $8,8 \mathrm{~nm}$ de $(\mathrm{AlGa}) \mathrm{As}$ non dopé, $[\mathrm{Al}]=0,33$; (v) $1 \mu \mathrm{m}$ de GaAs dopé à $2 \times 10^{17} \mathrm{~cm}^{-3}$, comme couche de contact.

Les caractéristiques $I(V)$ (Fig. 1) furent mesurées à $77 \mathrm{~K}$, l'échantillon étant monté sur un simple support de transistor. La courbe du haut sur la figure 1 fut obtenue sans condensateur extérieur aux bornes du dispositif et une région d'hystérésis est visible. Un oscilloscope connecté aux bornes du dispositif indique clairement la présence d'oscillations dans la zone de RDN. Connecter un condensateur aux bornes des fils émergeant du cryostat ne supprime pas les oscillations, cependant, lorsqu'un condensateur de $0,28 \mathrm{pF}$ est monté directement aux

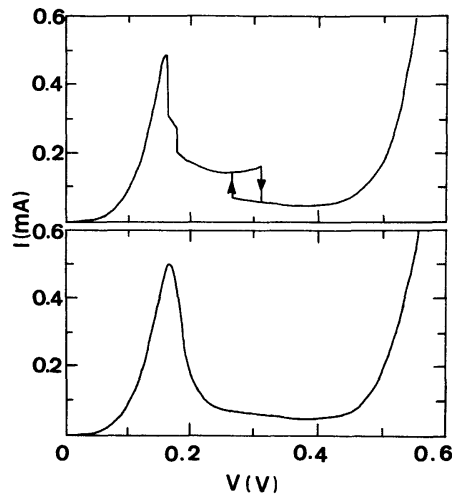

Fig. 1. - Caractéristiques courant-tension à $77 \mathrm{~K}$, d'un plot de $100 \mu \mathrm{m}$ sur la structure A (puits de 5,6 $\mathrm{nm}$ ) décrite dans le texte. La courbe du haut montre le courant lorsqu'aucun condensateur extérieur n'est branché aux bornes de l'échantillon. Dans la zone de tensions correspondant à la bistabilité, le dispositif oscille à haute fréquence. Dans la courbe du bas, les oscillations et la bistabilité ont été complètement supprimées par la connexion d'un condensateur de $0,28 \mathrm{pF}$ directement aux bornes de l'échantillon.

[The current-voltage characteristics at $77 \mathrm{~K}$ of a $100 \mu \mathrm{m}$ mesa fabricated from the double barrier structure A (56 $\AA$ well) described in the text. The upper curve shows the d.c. current with no external capacitor across the mesa. In the voltage range corresponding to current bistability, the device is oscillating at high frequency. In the lower curve, the oscillations and bistability are completely suppressed by placing a $0.28 \mu \mathrm{F}$ capacitor directly across the mesa.]

bornes du support de transistor, on obtient alors la courbe $I(V)$ stabilisée du bas sur la figure 1 .

Une seconde méthode peut permettre de stabiliser le circuit contre les oscillations. Il s'agit de connecter en parallèle avec l'échantillon, une petite résistance $r(\simeq 25 \Omega)$ de suffisamment faible impédance, de façon à ce que $r<|\mathrm{d} V / \mathrm{d} I|$ dans toute la zone de RDN. On obtient une courbe $I(V)$ stabilisée du dispositif en parallèle avec la résistance en appliquant une rampe de tension. La caractéristique $I(V)$ de l'échantillon seul est obtenue en soustrayant à la courbe précédemment mesurée, le courant $V / r$ circulant dans la résistance. La figure 2 montre le résultat classique obtenu pour un échantillon de $5 \mu \mathrm{m}$ de diamètre (structure $\mathrm{B}$ ) élaboré comme suit dans l'ordre de croissance à partir du substrat de $\mathrm{n}^{+} \mathrm{GaAs}$ :

(i) $1 \mu \mathrm{m}$ de GaAs dopé à $2 \times 10^{17} \mathrm{~cm}^{-3}$; (ii) $50 \mathrm{~nm}$ de GaAs dopé à $2 \times 10^{16} \mathrm{~cm}^{-3}$; (iii) $2,5 \mathrm{~nm}$ de GaAs non dopé; (iv) $5,6 \mathrm{~nm}$ de (AlGa)As non dopé, $[\mathrm{Al}]=0,44$; (v) $5 \mathrm{~nm}$ de GaAs non dopé ; (vi) $5,6 \mathrm{~nm}$ de (AlGa)As non dopé, $[\mathrm{Al}]=0,44$; (vii) $25 \mathrm{~nm}$ de GaAs non dopé ; (viii) $50 \mathrm{~nm}$ de GaAs dopé à $2 \times 10^{16} \mathrm{~cm}^{-3}$ et (ix) $0,5 \mu \mathrm{m}$ de GaAs dopé à $2 \times 10^{17} \mathrm{~cm}^{-3}$. 


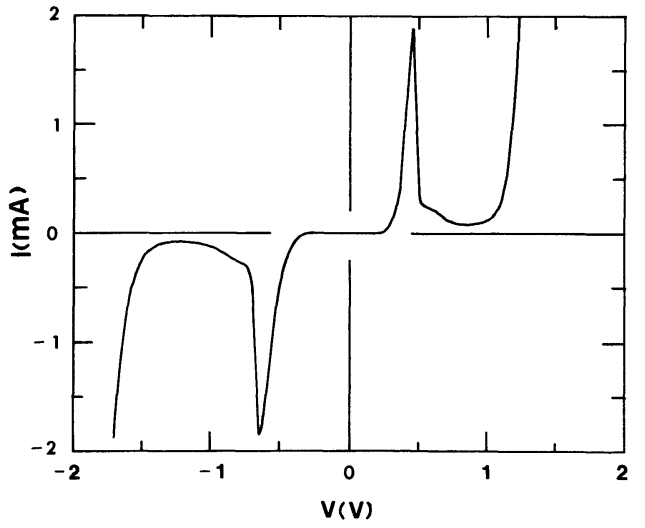

Fig. 2. - Caractéristiques courant-tension à $77 \mathrm{~K}$ d'un plot de $5 \mu \mathrm{m}$ sur la structure B (puits de $5 \mathrm{~nm}$ ) décrite dans le texte. Les oscillations et la bistabilité sont supprimées par la connection d'une faible résistance $(25 \Omega)$ en parallèle avec l'échantillon.

[The current-voltage characteristics at $77 \mathrm{~K}$ of a $5 \mu \mathrm{m}$ diameter mesa fabricated from structure B (50 $\AA$ well) described in the text. The oscillations and circuit bistability are suppressed by connecting a small resistor $(25 \Omega)$ in parallel with the device.]

Le rôle des couches non dopées de chaque côté des barrières est d'empêcher la diffusion d'impuretés dans la barrière et de diminuer les diffusions sur les impurités ionisées qui, comme nous le verrons plus tard ont tendance à diminuer le rapport pic/vallée de ces dispositifs.

Nous concluons donc que nos expériences confirment les objections relevées par Sollner concernant l'annonce par Goldman et al. de l'observation d'une bistabilité intrinsèque dans les structures à double barrière à effet tunnel résonnant. Cependant, l'étude des magnéto-oscillations du courant tunnel [3-5] montre clairement l'existence d'une accumulation de charges dans le puits aux tensions de résonance. De plus, l'existence d'une bistabilité intrinsèque entrâ̂née par l'effet électrostatique de ces charges reste théoriquement probable, et de récentes études expérimentales sur des structures asymétriques $[10,11]$ semblent de même en confirmer l'existence.

\section{Diffusions élastiques et inélastiques et le courant vallée.}

L'une ou l'autre des méthodes décrites ci-dessus pour stabiliser le circuit contre les oscillations nous permet d'étudier plus en détail le courant de ces dispositifs en présence d'un champ magnétique, ou encore la dynamique des électrons aux tensions audessus du pic principal de résonance. Comme le montrent les figures 1 et 2 , il existe dans cette région une petite bosse dans les caractéristiques $I(V)$. Goldman et al. [7] ont attribué cette particularité à la traversée par effet tunnel d'électrons, de l'émetteur au niveau lié du puits, assistée par l'émission de phonons optiques longitudinaux. Si un électron traverse les barrières sans collision, ce qui est supposé être le cas pour la majorité des électrons contribuant au pic principal de résonance, la composante du vecteur d'onde $k_{\perp}$, perpendiculaire à la direction du courant, est conservée. L'émission d'un phonon LO ou une diffusion élastique viole cette loi de conservation. D'autre part, la diffusion intersousbande due à l'émission de phonons $\mathrm{LO}$ a déjà été identifiée dans les structures à effet tunnel résonnant [8], à partir d'une analyse des magnéto-oscillations du courant tunnel. Cette bosse liée à l'émission de phonons LO, ainsi que la contribution au courant vallée des processus de diffusion élastique, peuvent être révélées plus clairement en appliquant un fort champ magnétique $\mathbf{B} / / \mathbf{J}$. En l'absence de champ magnétique, $k_{\perp}$ est conservé si un électron ne subit aucune diffusion au cours de son passage dans la barrière. En présence d'un champ magnétique intense, l'énergie cinétique dans le plan des barrières est quantifiée et l'énergie totale de l'électron dans la zone d'accumulation de l'émetteur et dans le puits est donnée par :

Emetteur

$$
E=E_{0}+(n+1 / 2) \hbar \omega_{\mathrm{c}}
$$

Puits quantique

$$
E=E_{1}+\left(n^{\prime}+1 / 2\right) \hbar \omega_{\mathrm{c}}
$$

où $n\left(n^{\prime}\right)$ est l'indice du niveau de Landau, $\omega_{\mathrm{c}}=e B / \mathrm{m}^{*}$, et $E_{0}$ et $E_{1}$ sont les niveaux liés fondamentaux de l'émetteur et du puits respectivement. La conservation de $k$ pour $B=0$ correspond en présence d'un champ magnétique à la conservation de l'indice du niveau de Landau, i.e., $p=n^{\prime}-n=0$, de façon à ce que dans les deus cas la résonance intervient à une tension pour laquelle $E_{0}=E_{1}$.

La figure 3 montre l'effet d'un champ magnétique B//J sur la caractéristique $I(V)$ d'un échantillon de $100 \mu \mathrm{m}$ de diamètre dont la largeur du puits quantique est de $117 \AA$ (structure C). A champ magnétique nul, les oscillations dans la zone de RDN sont supprimées en connectant une résistance de $25 \Omega$ aux bornes de l'échantillon. La structure de celui-ci est, dans l'ordre de croissance à partir du substrat de $\mathrm{n}^{+} \mathrm{GaAs}$ :

(i) $2 \mu \mathrm{m}$ de GaAs dopé à $2 \times 10^{18} \mathrm{~cm}^{-3}$; (ii) $50 \mathrm{~nm}$ de GaAs dopé à $2 \times 10^{16} \mathrm{~cm}^{-3}$; (iii) $2,5 \mathrm{~nm}$ de $\mathrm{GaAs}$ non dopé ; (iv) $5,6 \mathrm{~nm}$ de (AlGa)As non dopé, $[\mathrm{Al}]=0,44$; (v) $11.7 \mathrm{~nm}$ de GaAs non dopé; (vi) $5,6 \mathrm{~nm}$ de (AlGa)As non dopé, $[\mathrm{Al}]=0,44$; (vii) $25 \mathrm{~nm}$ de GaAs non dopé ; (viii) $50 \mathrm{~nm}$ de GaAs dopé à $2 \times 10^{16} \mathrm{~cm}^{-3}$ et (ix) $0,5 \mu \mathrm{m}$ de GaAs dopé à $2 \times 10^{18} \mathrm{~cm}^{-3}$.

La figure 3 montre uniquement la partie des caractéristiques $I(V)$ correspondant aux faibles ten- 


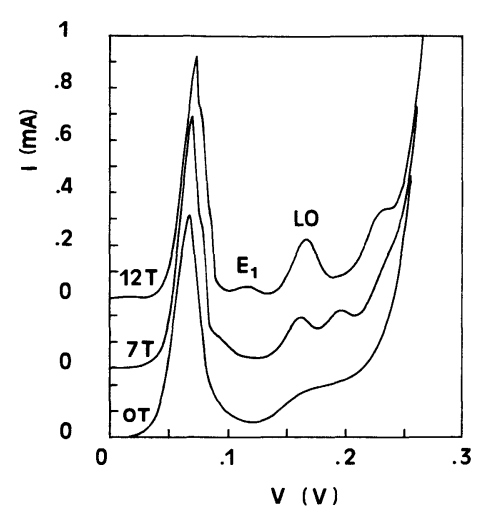

Fig. 3. - Caractéristiques courant-tension à $4 \mathrm{~K}$ et pour plusieurs champs magnétiques $\mathbf{B} / / \mathbf{J}$, d'un plot de $100 \mu \mathrm{m}$ de diamètre sur la structure $C$ (puits de $11,7 \mathrm{~nm}$ ) décrite dans le texte. Les courbes montrent uniquement la région correspondant à la première résonance. Les oscillations et la bistabilité sont supprimées par la connexion d'une faible résistance $(25 \Omega)$ en parallèle avec l'échantillon.

[The current-voltage characteristics at $4 \mathrm{~K}$ and at various magnetic fields $\mathbf{B} / / \mathbf{J}$ of a $100 \mu \mathrm{m}$ diameter mesa fabricated from structure $C$ (117 $\AA$ well) described in the text. The curves show only the region of the first resonance. The oscillations and current bistability are suppressed by a small resistor $(25 \Omega)$ in parallel with the device.]

sions. D'autres pics sont en effet observés dans le courant à $420 \mathrm{mV}$ et $890 \mathrm{mV}$, ceux-ci correspondant à la résonance via le deuxième et troisième niveau lié du puits. On notera essentiellement les particularités suivantes sur la figure 3 :

premièrement, le champ magnétique accroît l'amplitude du pic correspondant aux phonons LO ;

deuxièmement, un second pic assez faible émerge du pic principal lorsque l'on augmente le champ magnétique. D'autres pics du même type évoluent de la même façon à partir du pic lié aux phonons LO ;

troisièmement, le champ magnétique accroît le rapport pic/vallée.

La figure 4 montre l'évolution des pics dans la caractéristique $I(V)$ en fonction du champ magnétique. Les transitions pour lesquelles $k_{\perp}$ (ou bien $n$ ) n'est pas conservé sont gouvernées uniquement par la règle de conservation de l'énergie. Pour les diffusions élastiques et l'émission de phonons LO, on a donc

$$
E_{0}=E_{1}+p \hbar e B / m^{*}\left(+\hbar \omega_{\mathrm{L}}\right) .
$$

$E_{0}-E_{1}$ augmente approximativement linéairement avec la tension appliquée à partir de sa valeur initiale (négative) à tension nulle. De plus, la figure 4 montre deux groupes distincts de lignes. A la limite $B \rightarrow 0$, le pic $E_{1}$ se confond au pic principal de résonance à $70 \mathrm{mV}$. Les pics $\mathrm{LO}_{\mathrm{p}}$ rejoignent le pic satellite du phonon LO à $160 \mathrm{mV}$. Le pic marqué $E_{1}$ correspond à l'effet tunnel non résonnant compre-

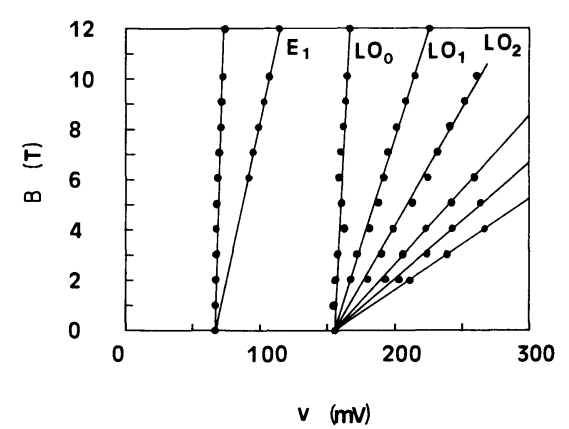

Fig. 4. - Diagramme montrant la dépendance en champ magnétique des pics dans les caractéristiques $I(V)$ de la figure 3. Les processus élastiques et inélastiques de diffusion donnant lieu à ces pics sont présentés dans le texte.

[Fan chart showing the magnetic field dependence $(\mathbf{B} / / \mathbf{J})$ of the peaks in $I(V)$ shown in figure 3. The elastic and inelastic (LO phonon) scattering processes giving rise to the oscillations are discussed in the text.]

nant une transition du niveau de Landau $n=0$ dans l'émetteur au niveau de Landau $n=1$ dans le puits, induite par un processus élastique (ou quasi élastique) de diffusion. Une telle transition pourrait être induite par les impuretés ionisées ou les rugosités d'interface (élastiques) ou encore par l'émission de phonons acoustiques (quasi élastique). Le pic principal de résonance qui ne bouge pratiquement pas avec le champ magnétique, correspond aux processus tunnels pour lesquels $n$ est conservé. Les pics marqués $\mathrm{LO}_{\mathrm{p}}$ correspondent aux transitions du niveau de Landau $n=0$ de l'émetteur au niveau de Landau $\mathrm{p}$ du puits, accompagné de l'émission d'un phonon LO.

Il est intéressant de noter que l'effet d'un fort champ magnétique est de supprimer les transitions induites par les processus (quasi-)élastiques de diffusion, pour certaines tensions (au-dessus et au-dessous de $E_{1}$ ) et de favoriser celles-ci à d'autres tensions (à $E_{1}$ ). A champ magnétique nul, de tels processus qui changent $k_{\perp}$ sont énergétiquement permis pour toute tension après le pic principal de résonance. A de telles tensions, le niveau lié du puits est au-dessous de l'énergie des électrons de l'émetteur. Cependant, un fort champ magnétique quantifie l'énergie des électrons dans le plan des couches, entraînant des pics fins dans les densités d'états. A certaines tensions (pour lesquelles les échelles de Landau ne sont pas juxtaposées) et en présence d'un fort champ magnétique, la loi de conservation de l'énergie annihile les diffusions (quasi-)élastiques dans le puits. Ceci explique donc l'accroissement du rapport pic/vallée avec le champ magnétique.

La différence en tension entre le pic principal et le pic lié à l'émission d'un phonon LO, est considérablement plus grande que l'énergie du phonon optique longitudinal dans $\mathrm{GaAs}(36 \mathrm{meV})$. Ceci est en accord avec la distribution de la chute de potentiel 
attendue dans ce dispositif : dans l'échelle de tension allant de 100 à $300 \mathrm{mV}$ seulement $30 \%$ de la tension totale appliquée sont distribués dans la zone d'accumulation de l'émetteur, la barrière de l'émetteur et la moitié du puits. Le reste est distribué entre l'autre moitié du puits, la barrière côté collecteur, et la zone de déplétion du collecteur.

\section{Etats quantifiés électriquement et magnétiquement dans des puits larges.}

Dans cette section nous étudions l'effet tunnel résonnant dans des structures à double barrière dont les puits sont très larges (60 à $120 \mathrm{~nm})$. Ces structures (D et $\mathrm{E}$ ) sont élaborées comme suit dans l'ordre de croissance à partir du substrat $\mathrm{n}^{+} \mathrm{GaAs}$ :

(i) $2 \mu \mathrm{m}$ de GaAs dopé à $2 \times 10^{18} \mathrm{~cm}^{-3}$; (ii) $50 \mathrm{~nm}$ de GaAs dopé à $2 \times 10^{16} \mathrm{~cm}^{-3}$; (iii) $2,5 \mathrm{~nm}$ de GaAs non dopé ; (iv) $5,6 \mathrm{~nm}$ de (AlGa)As non dopé, $[\mathrm{Al}]=0,44$; (v) 60(120) nm de GaAs non dopé pour la structure $\mathrm{D}$ (structure $\mathrm{E})$; (vi) 5,6 nm de (AlGa)As non dopé, $[\mathrm{Al}]=0,44$; (vii) $25 \mathrm{~nm}$ de GaAs non dopé ; (viii) $50 \mathrm{~nm}$ de GaAs dopé à $2 \times 10^{16} \mathrm{~cm}^{-3}$ et (ix) $0,5 \mu \mathrm{m}$ de GaAs dopé à $2 \times 10^{18} \mathrm{~cm}^{-3}$.

La figure 5 montre les caractéristiques $I(V)$ de la structure $\mathrm{D}$ obtenues à $4 \mathrm{~K}$. On peut remarquer que le courant est tracé logarithmiquement sur 5 ordres de grandeurs. On observe jusqu'à seize zones distinctes de RDN jusqu'à $1 \mathrm{~V}$, et d'autres structures attribuables à l'effet tunnel via les niveaux virtuels au-dessus de la seconde barrière sont observables clairement aux plus fortes tensions. L'existence d'états stationnaires résonnants bien définis suppose que les électrons parcourent une distance égale à

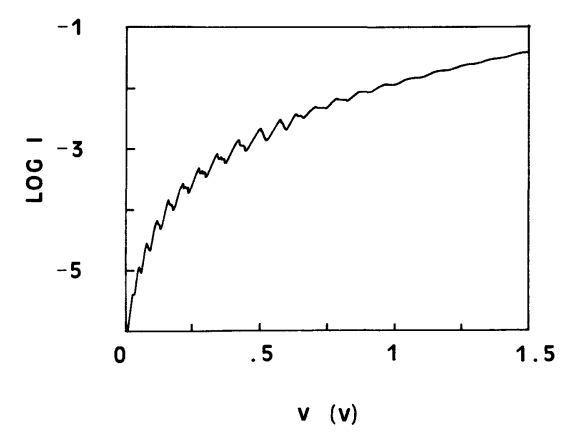

Fig. 5. - Logarithme du courant en fonction de la tension à $4 \mathrm{~K}$ pour un plot de $100 \mu \mathrm{m}$ sur la structure $\mathrm{D}$ (puits de $60 \mathrm{~nm}$ ) décrite dans le texte. On observe clairement jusqu'à 16 régions de RDN attribuables aux résonances via les niveaux liés très resserrés en énergie du puits quantique.

[The current-voltage characteristics at $4 \mathrm{~K}$ (I is plotted logarithmically) of a $100 \mu \mathrm{m}$ diameter mesa fabricated from the double barrier structure D (600 $\AA$ well) described in the text.] deux fois la largeur du puits (120 nm pour structure $D$ et 240 pour structure $E$ !), sans subir énormément de diffusions, même lorsque les électrons sont injectés dans le puits avec une énergie cinétique de plusieurs centaines de meV.

De telles structures sont parfaitement appropriées pour étudier l'effet d'un potentiel de confinement sur les états propres électroniques dans des champs électriques et magnétiques croisés $\mathbf{B} \perp \mathbf{E}$. Le champ électrique étant perpendiculaire aux barrières, nous appliquerons le champ magnétique dans le plan des couches. La figure 6 montre l'effet d'un champ $\mathbf{B} \perp \mathbf{J}$ sur les caractéristiques courant-tension. De façon à augmenter la résolution des résonances, nous utilisons la dérivée seconde du courant par rapport à la tension $\mathrm{d}^{2} I / \mathrm{d} V^{2}$. A forts champs, on voit que les résonances se partagent en deux groupes distincts, la flèche sur la figure 6 indique la séparation dans le cas d'un champ magnétique $B=7 \mathrm{~T}$.

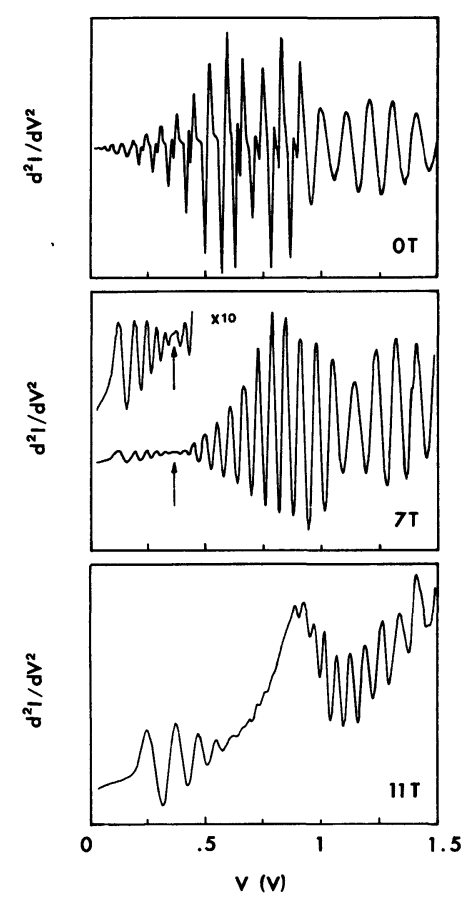

Fig. 6. - Tracé de $\mathrm{d}^{2} I / \mathrm{d} V^{2}$ en fonction de $V$ pour la structure $\mathrm{D}$ à $4 \mathrm{~K}$ pour plusieurs champs magnétiques, B $\perp \mathbf{J}$. Pour $B \geqslant 2 \mathrm{~T}$, on observe une série d'oscillations due à l'effet tunnel sur les états quantifiés magnétiquement d'interfaces. Pour la courbe à $7 \mathrm{~T}$, la flèche indique la tension critique $V_{\mathrm{c}}$ de disparition des orbites d'interface.

[Plot of $\mathrm{d}^{2} I / \mathrm{d} V^{2}$ versus $V$ for structure $\mathrm{D}(600 \AA$ well) at $4 \mathrm{~K}$ for various magnetic fields, $\mathrm{B} \perp \mathrm{J}$. At zero and low magnetic fields the oscillatory structure can be attributed to electrons tunnelling into electrically quantised bound states of the well and into " above the barrier » resonances of the well. For $B \geqslant 2 \mathrm{~T}$, an additionnal series of oscillations due to tunnelling into magnetically quantised interface states can be observed at low voltages. For the trace at $7 \mathrm{~T}$, the critical voltage $V_{c}$ for the disappearance of the skipping orbits is indicated by an arrow.] 
A faible champ magnétique, les orbites cyclotrons butent contre les barrières de chaque côté du puits, mais à forts champs magnétiques, des orbites cyclotrons se développent à l'interface, qui ne viennent rebondir de façon répétée que contre une seule interface [12]. En outre, on remarquera que de telles orbites d'interfaces ont déjà été mises en évidence dans les hétérostructures à simple barrière [9], mais celles-ci se situaient dans une région fortement dopée, où le champ électrique était à vrai dire nul. Dans le cas présent, il existe un fort champ électrique dans le puits où ces orbites se développent. La trajectoire de l'électron est alors de type cycloïdal, avec une vitesse d'entraînement uniforme $v_{\mathrm{d}}=E / B$ parallèle à l'interface, superposée à une trajectoire circulaire, de vitesse angulaire $\omega_{\mathrm{c}}=e B / \mathrm{m}^{*}$ dans le plan perpendiculaire à $\mathbf{B}$.

Nous supposons que les électrons traversant la barrière de l'émetteur, arrivent dans le puits avec une énergie cinétique faible comparée à l'énergie cinétique gagnée au cours de la trajectoire cycloïdale qui s'ensuit. La composante circulaire de la trajectoire cycloïdale a alors un rayon $R=v_{\mathrm{d}} / \omega_{\mathrm{c}}$ le centre de l'orbite étant lui, situé à la même distance $R$ de la barrière côté émetteur. La largeur d'une telle orbite dans la direction du champ électrique est donnée par $2 R=2 v_{\mathrm{d}} / \omega_{\mathrm{c}}=2 m^{*} E / e B^{2}$. Le cas critique où l'orbite cycloïde touche juste la barrière du collecteur, est donc donné par $2 R=w$, où $w$ est la largeur du puits. On pourrait alors prendre $E=V / d$, où $d=w+2 b+\lambda_{\mathrm{L}}+\lambda_{\mathrm{R}}$ avec $\lambda_{\mathrm{L}}$ et $\lambda_{\mathrm{R}}$ étant approximativement les longueurs d'écran pour l'émetteur et le collecteur respectivement. Ainsi, l'effet tunnel sur ces états cycloïdaux d'interfaces, n'interviennent que pour des tensions $V<V_{\mathrm{c}}$, où la tension critique $V_{\mathrm{c}}=e B^{2} w d / 2 m^{*}$. Cette dépendance en $B^{2}$ est observée expérimentalement. Pour $B=7 \mathrm{~T}$, avec $\lambda_{\mathrm{L}}=\lambda_{\mathrm{R}} \simeq 10 \mathrm{~nm} \quad\left(d=91 \mathrm{~nm}, \quad m^{*}=0,067 m_{\mathrm{e}}\right)$ nous trouvons $V_{\mathrm{c}}=350 \mathrm{mV}$. Cette valeur est indiquée par une flèche sur la figure 6 et se situe bien à la séparation entre le groupe de résonances liées aux états cycloïdaux ne faisant intervenir des réflexions que sur une interface $\left(V<V_{\mathrm{c}}\right)$, et le groupe de résonances liées aux états pour lesquels la trajectoire électronique est restreinte par les réflexions sur les interfaces des deux côtés du puits $\left(V>V_{\mathrm{c}}\right)$.

Nous avons analysé les positions des résonances (minima de la courbe $\mathrm{d}^{2} I / \mathrm{d} V^{2}$ en fonction de $V$ ) liées aux états cycloïdaux d'interfaces sur une large gamme de champs magnétiques et de tensions.

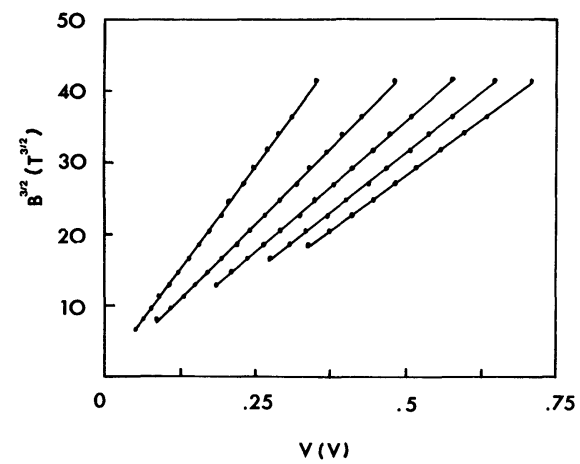

Fig. 7. - Dépendances en $B^{3 / 2}$ des positions en tension des résonances liées aux états cycloïdaux d'interface correspondant à $n=0,1,2,3,4$ (de gauche à droite).

$\left[B^{3 / 2}\right.$ dependence of voltage positions of resonances due to skipping states corresponding to $n=0,1,2,3,4$ (from left to right).]

Lorsque l'on trace ces positions sur un diagramme $B^{3 / 2}=f(V)$, on obtient les dépendances linéaires de la figure 7 . Ces résultats peuvent d'expliquer en termes de quantification des orbites cycloïdales dont on a parlé ci-dessus. L'énergie de la trajectoire circulaire, $m^{*} \omega_{\mathrm{c}}^{2} R^{2} / 2$, lorsqu'elle est quantifiée, prend les valeurs permises $(n+\phi) \hbar \omega_{c}$, où le facteur de phase $\phi$ vaur 3/4 dans l'approximation WKB [13]. En remplaçant $R$ par $v_{\mathrm{d}} / \omega_{\mathrm{c}}$, on obtient $m^{*} v_{\mathrm{d}}^{2} / 2=(n+\phi) \hbar \omega_{\mathrm{c}}$, ce qui peut s'écrire

$$
m^{* 2} E^{2} / 2 e \hbar B^{3}=n+\phi \quad n=0,1, \ldots
$$

Ceci signifie que pour une tension donnée ( $E$ fixé), les résonances sont périodiques en $1 / B^{3}$. (1) est en accord avec la variation linéaire de $B^{3 / 2}$ avec $V$ donnée par la figure 7. D'autre part, en utilisant le facteur de phase $\phi$ donné par l'approximation WKB, la théorie est en accord avec les résultats expérimentaux à $15 \%$ près, ce qui est satisfaisant si l'on considère l'approximation faite d'une faible énergie cinétique de départ et l'incertitude sur la distance $\mathrm{d}$ sur laquelle est distribuée la tension appliquée.

\section{Remerciements.}

Les auteurs tiennent à remercier le Conseil Régional Midi-Pyrénées ainsi que la Commision de la Communauté Européenne pour leurs soutiens financiers. 


\section{References}

[1] Goldman V. J., Tsui D. C. et Cunningham J. E., Phys. Rev. Lett. 58 (1987) 1256 ; ibid. 59 (1987) 1623.

[2] Sollner T. C. L. G., Phys. Rev. Lett. 59 (1987) 1622.

[3] Payling C. A., Alves E. S., Eaves L., Foster T. J., Henini M., Hughes O. H., Simmonds P. E., Portal J. C., Sheard F. W. et ToOmbs G. A., Proc. 7th Int. Conf. on the Electronic Properties of Two Dimensional Systems, Santa Fe, 1987. A paraître dans Surf. Sci. (1988). Voir aussi Payling C. A., Alves E. S., Eaves L., Foster T. J., Henini M., Hughes O. H., SiMmonds P. E., Portal J. C., Hill G. et Pate M. A., Proc. 3rd Int. Conf. on Modulated Semiconductor Structures, Montpellier, 1987. Paru dans J. Phys. Colloq. France C 5 (1987) 289.

[4] Toombs G. A., Alves E. S., Eaves L., Foster T. J., Henini M., Hughes O. H., LEAdBEATER M. L., Payling C. A., Sheard F. W., Claxton P. A., Hill G., Pate M. A. et Portal J. C., 14th Int. Symposium on Gallium Arsenide and Related Compounds, Crete (1987).

[5] Goldman V. J., Tsui D. C. et Cunningham J. E., Phys. Rev. B 35 (1987) 9387.

[6] Sheard F. W. et Toombs G. A., Appl. Phys. Lett. 52 (1988) 1228.

[7] Goldman V. J., Tsui D. C. et Cunningham J. E.,
Phys. Rev. B 36 (1987) 7635. Voir aussi Proc. 3rd Int. Conf. on Modulated Semiconductor Structures, Montpellier, 1987. J. Phys. Colloq. France C 5 (1987) 467.

[8] Eaves L., Toombs G. A., Sheard F. W., Payling C. A., Leadbeater M. L., Alves E. S., FosTER T. J., SIMMONDS P. E., HENINI M. et Hughes O. H., Appl. Phys. Lett. 52 (1988) 212.

[9] Snell B. R., Chan K. S., Sheard F. W., Eaves L., Toombs G. A., Maude D. K., Portal J. C., Bass S. J., Claxton P., Hill G. et Pate M. A., Phys. Rev. Lett. 59 (1987) 2806.

[10] Leadbeater M. L., Alves E. S., Eaves L., Henini M., Hughes O. H., Sheard F. W., TOOMBS G. A., à paraitre dans Proceedings of the Fourth Int. Conf. on Superlattices, Microstructures and Microdevices, Trieste (1988).

[11] Zaslavsky A., Goldman V. J., Tsui D. C., CunNINGHAM J. E., à paraître dans A.P.L., octobre 1988.

[12] Dans ce cas le potentiel de confinement est constitué d'un côté d'un potentiel « dur » qu'est l'interface barrière émettrice-puits, et de l'autre d'un potentiel «mou », qu'est le potentiel quadratique dû au champ magnétique. Ceci justifie l'appellation « états cycloïdaux d'interface » utilisée dans le texte.

[13] Landau et Lifchitz, Mécanique Quantique, Ed. MIR, 196. 